\title{
Coexistence between silent and bursting states in a biophysical Hodgkin-Huxley-type
} of model

Stankevich, Nataliya; Mosekilde, Erik

Published in:

Chaos

Link to article, DOI:

$10.1063 / 1.4986401$

Publication date:

2017

Document Version

Publisher's PDF, also known as Version of record

Link back to DTU Orbit

Citation (APA):

Stankevich, N., \& Mosekilde, E. (2017). Coexistence between silent and bursting states in a biophysical Hodgkin-Huxley-type of model. Chaos, 27(12), [123101]. https://doi.org/10.1063/1.4986401

\section{General rights}

Copyright and moral rights for the publications made accessible in the public portal are retained by the authors and/or other copyright owners and it is a condition of accessing publications that users recognise and abide by the legal requirements associated with these rights.

- Users may download and print one copy of any publication from the public portal for the purpose of private study or research.

- You may not further distribute the material or use it for any profit-making activity or commercial gain

- You may freely distribute the URL identifying the publication in the public portal

If you believe that this document breaches copyright please contact us providing details, and we will remove access to the work immediately and investigate your claim. 


\section{Coexistence between silent and bursting states in a biophysical Hodgkin-Huxley-type of model}

Nataliya Stankevich, and Erik Mosekilde

Citation: Chaos 27, 123101 (2017);

View online: https://doi.org/10.1063/1.4986401

View Table of Contents: http://aip.scitation.org/toc/cha/27/12

Published by the American Institute of Physics

\section{Articles you may be interested in}

Observability and synchronization of neuron models

Chaos: An Interdisciplinary Journal of Nonlinear Science 27, 103103 (2017); 10.1063/1.4985291

Synchronous behaviour of two interacting oscillatory systems undergoing quasiperiodic route to chaos

Chaos: An Interdisciplinary Journal of Nonlinear Science 27, 103119 (2017); 10.1063/1.4991744

Exploring the route to measure synchronization in non-linearly coupled Hamiltonian systems

Chaos: An Interdisciplinary Journal of Nonlinear Science 27, 113103 (2017); 10.1063/1.4996814

Insensitivity of synchronization to network structure in chaotic pendulum systems with time-delay coupling

Chaos: An Interdisciplinary Journal of Nonlinear Science 27, 126702 (2017); 10.1063/1.5010304

Attractors in complex networks

Chaos: An Interdisciplinary Journal of Nonlinear Science 27, 103105 (2017); 10.1063/1.4996883

Dissipative structures, machines, and organisms: A perspective

Chaos: An Interdisciplinary Journal of Nonlinear Science 27, 104607 (2017); 10.1063/1.5001195

Welcome to a Smarter Search $\mathbf{Q}$ with the redesigned Physics Today Buyer's Guide 


\title{
Coexistence between silent and bursting states in a biophysical Hodgkin-Huxley-type of model
}

\author{
Nataliya Stankevich ${ }^{1,2,3, a)}$ and Erik Mosekilde ${ }^{4}$ \\ ${ }^{1}$ Department of Radio-Electronics and Telecommunications, Yuri Gagarin State Technical University \\ of Saratov, 77, Politechnicheskaya, Saratov 410054, Russian Federation \\ ${ }^{2}$ Faculty of Information Technology, University of Jyväskylä, P.O. Box 35, FI-40014 Jyväskylä, Finland \\ ${ }^{3}$ Faculty of Mathematics and Mechanics, St. Petersburg State University, 28, Peterhof, Universitetskiy proezd, \\ St. Petersburg 198504, Russian Federation \\ ${ }^{4}$ Department of Physics, Technical University of Denmark, 2800 Lyngby, Denmark
}

(Received 5 June 2017; accepted 9 November 2017; published online 1 December 2017)

\begin{abstract}
Classification of the dynamical mechanisms that support bistability between bursting oscillations and silence has not yet been clarified in detail. The purpose of this paper is to demonstrate that the coexistence of a stable equilibrium point with a state of continuous bursting can occur in a slightly modified, biophysical model that describe the dynamics of pancreatic beta-cells. To realize this form of coexistence, we have introduced an additional voltage-dependent potassium current that is activated in the region around the original, unstable equilibrium point. It is interesting to note that this modification also leads the model to display a blue-sky catastrophe in the transition region between chaotic and bursting states. Published by AIP Publishing.
\end{abstract}

https://doi.org/10.1063/1.4986401

\begin{abstract}
Multistability is a characteristic feature of many types of cells, neural networks, and other forms of oscillatory biophysical systems. This feature is particularly significant in connection with the study of interacting ensembles of many, nearly identical subsystems. It is well-known that, for instance, a variety of unusual phenomena that emerge in ensembles of coupled oscillators can lead to significant reconstructions of an oscillator population, and the presence of coexisting states can significantly influence on the dynamics of all ensemble. In this paper, we introduce a modified version of the well-known model describing dynamics of pancreatic beta-cells obtained by introducing a new type of potassium-like ion channel with a characteristic set of channel parameters selected in accordance with a standard Hodgkin-Huxley formalism. The suggested modification is designed to be local and small enough to allow for the existence of an attracting state inside the regime of stable bursting dynamics. This provides for the presence of multistability in the modified beta-cell model and, at the same time, serves as an example of a biophysical system that allows for the coexistence of a stable equilibrium point with large amplitude bursting.
\end{abstract}

\section{INTRODUCTION}

Multistability, or coexistence of dynamical regimes, is a characteristic feature of many types of cells, neural networks, ${ }^{1-4}$ and other forms of oscillatory biophysical systems. ${ }^{5-11}$ This feature is particularly significant in connection with the study of interacting ensembles of many, nearly

\footnotetext{
a) Author to whom correspondence should be addressed: stankevichnv@ mail.ru
}

identical subsystems. It is well-known that, for instance, a variety of unusual phenomena that emerge in ensembles of coupled oscillators can lead to the overall reconstruction of the oscillator population or to its total collapse. It is broadly accepted that, for instance, synchronization plays an important role in the pathogenesis of neurological diseases such as Parkinson's disease and essential tremor ${ }^{10-12}$ and, more specifically, Parkinson's disease is associated with asynchronous pacemaker activity involving a population of many thousands of neurons in the basal ganglia. ${ }^{13,14}$ Another fundamental phenomenon that can emerge in ensembles of coupled system is oscillator quenching. ${ }^{15}$ The biomedical significance of this phenomenon again hinges on the fact that the suppression or disruption of oscillations is involved in the treatment of a variety of neuronal disorders such as Alzheimer's and Parkinson's disease.

In this context, the coexisting states of the single element of the system play a significant role. Special attention should be paid to hidden or rare attractors. In line with the recent survey papers by Leonov and Kuznetsov et al., ${ }^{16-18}$ an attracting state may be classified as either "hidden" or "self-excited," with hidden attractors representing all such attractors that do not connect to a stable equilibrium state. The presence of hidden attractors in a system is associated with multistability, and in some cases finding of hidden or rare attractor may be a challenging task, but it can influences the dynamics of the integrated system dramatically.

At present, the mechanisms that allow bistability, such as the coexistence of tonic spiking and silence or the coexistence of tonic spiking and bursting, appear to be relatively well described. On the other hand, the dynamical mechanisms that support bistability between bursting and silence have not yet been examined to the same extent. ${ }^{4}$ Classification of mechanisms that support the coexistence of oscillatory and 
silent regimes is so far incomplete, and this remains a challenge both in relation to the progress of dynamical systems theory and in relation to important issues in neuroscience. An example of a system that displays coexistence of silence and bursting oscillations was presented in the recent paper by Malashchenko et al.. ${ }^{4}$ Yet, further attention to this problem is clearly desirable.

This study takes a point of departure in the classic Sherman model that has been widely used to describe the spiking and bursting dynamics of insulin-secreting pancreatic beta-cells, ${ }^{19,20}$ and also this model is widely used for description of the dynamics of neuron. Experimentally, one can observe, for instance, how the secretion of insulin increases with the fraction of time that the cells spend in the spiking state. On the other hand, the duration of the silent phase between two bursts is regulated by the rate at which calcium is removed from the interior of the cell. The spiking oscillations typically display a period of $1-10 \mathrm{~s}$, whereas the duration of the bursting period typically varies from 0.2 to $5.0 \mathrm{~min}$. It is interesting to note that, however, individual beta-cells do not burst. ${ }^{21,22}$ The opening probability for the potassium channels is too small for the individual cell to present a regular spiking signal and, only in the form of clusters of 30-100 synchronized cells, regular spiking oscillations will occur. Both electrical coupling and so-called glucose sensing are involved in the cellular synchronization.

We have previously performed a variety of one- and two-dimensional bifurcation analyses ${ }^{21}$ for the considered beta-cell model ${ }^{23}$ and demonstrated how the dynamics of this system after an initial Hopf bifurcation may display an unusual structure of overlapping resonance tongues. We have also outlined the main bifurcation structure for a model of two coupled, identical pancreatic cells ${ }^{24}$ and we have described an interesting form of phase synchronization that can occur when the uncoupled oscillators can synchronize in a variety of different configurations. ${ }^{25}$ Most recently, we have contributed to a study of the co-existence of hidden attractors in a variety of different systems, ${ }^{26}$ and we used fluorescence microscopy to demonstrate how externally forced glucose oscillations can induce distinct $1: 1$ and 1:2 entrainment of oscillations in islet $\mathrm{Ca}^{2+}$ concentrations and mitochondrial membrane potential. ${ }^{27}$

In this paper, we introduce a modified version of the Sherman model obtained by introducing a new type of potassium-like ion channel with a characteristic set of channel parameters selected in accordance with a standard Hodgkin-Huxley formalism. The suggested modification is designed to be local and small enough to allow for the existence of an attracting state inside the regime of stable bursting dynamics. This provides for the presence of multistability in the modified beta-cell model and, at the same time, serves as an example of a biophysical system that allows for the coexistence of a stable equilibrium point with large amplitude bursting. Besides the possibility of observing hidden attractors in a type of beta-cell like model, our interest in the considered system originates from the possibility of observing processes such as the intracellular dynamics of $\mathrm{Ca}^{2+}$-sensitive $\mathrm{K}^{+}$-channel dynamics ${ }^{28}$ or the effect of alternative controls on the cell dynamics. ${ }^{29} \mathrm{We}$ first present the structure of the original model and describe some of the characteristic bifurcation structure of that model. Hereafter follows a description of the modified cell model and the associated characteristic phenomena. Finally, in the last section, we discuss the modified beta cell model in the light of its underlying biological mechanisms.

\section{PRESENTATION OF THE BURSTING MODEL}

It is well known that the electrical activity of pancreatic beta-cells and other biological cells relies on a number of different types of voltage- and ligand-gated ion channels that are permeable to inorganic ions such as sodium, potassium, chloride, and calcium. Increasing evidence suggests that ion channels not only regulate membrane potential, ion homeostasis, and electrical signaling of these cells but also play an important role in cell proliferation, migration, apoptosis, and differentiation. Recently, the role of ion channels in different oncogenic processes was demonstrated. ${ }^{30-32}$

Over the years, studies of complex pancreatic systems have been performed on individual beta-cells from mice, on small and larger groups of interacting beta-cells, and on clusters of interacting islets. Together these studies have contributed significantly to a better understanding of the role of the inhomogeneity that exists at different levels of the interaction including, for instance, the more or less random mix of different cell types across the pancreatic tissue, the anisotropy associated with the preferential arrangement of the beta-cells along the pancreatic blood vessels, and the variation of the tissue properties along the edges of the islets. However, as mentioned above, only reasonably large and uniform clusters of pancreatic beta-cells display the characteristic bursting dynamics with its alternation between of rapid spiking and periods of silence.

As the starting point for our analysis, let us use the following simplified pancreatic beta-cell model based on the Hodgkin-Huxley formalism as suggested by Sherman et al. ${ }^{20}$

$$
\begin{aligned}
\tau \dot{V} & =-I_{C a}(V)-I_{K}(V, n)-I_{S}(V, S), \\
\tau \dot{n} & =\sigma\left(n_{\infty}(V)-n\right), \\
\tau_{S} \dot{S} & =S_{\infty}(V)-S .
\end{aligned}
$$

Here, $V$ represents the membrane potential, $n$ may be interpreted as the opening probability of the potassium channels, and $S$ accounts for the presence of a slow variable in the system. As previously noted, the precise biophysical interpretation of this variable remains unclear. The variables $I_{C a}(V)$ and $I_{K}(V, n)$ are the calcium and potassium currents, $g_{C a}=3.6$ and $g_{K}=10.0$ are the associated conductances, and $V_{C a}=25 \mathrm{mV}$ and $V_{K}=-75 \mathrm{mV}$ are the respective Nernst (or reversal) potentials. Together with $I_{S}(V, S)$, the slow calcium current $I_{C a}$ and the potassium current $I_{K}$ define the three transmembrane currents of our basic system

$$
\begin{gathered}
I_{C a}(V)=g_{C a} m_{\infty}(V)\left(V-V_{C a}\right), \\
I_{K}(V, n)=g_{K} n\left(V-V_{K}\right), \\
I_{S}(V, n)=g_{S} S\left(V-V_{K}\right),
\end{gathered}
$$


TABLE I. Parameters for model (1). ${ }^{18}$

\begin{tabular}{lcc}
\hline \hline$\tau=0.02 \mathrm{~s}$ & $\tau_{\mathrm{S}}=35 \mathrm{~s}$ & $\sigma=0.93$ \\
$g_{\mathrm{Ca}}=3.6$ & $g_{\mathrm{K}}=10.0$ & $g_{\mathrm{S}}=4.0$ \\
$V_{\mathrm{Ca}}=25.0 \mathrm{mV}$ & $V_{\mathrm{K}}=-75 \mathrm{mV}$ & \\
$\theta_{\mathrm{m}}=12.0 \mathrm{mV}$ & $\theta_{\mathrm{n}}=5.6 \mathrm{mV}$ & $\theta_{\mathrm{S}}=10.0 \mathrm{mV}$ \\
$V_{\mathrm{m}}=-20.0 \mathrm{mV}$ & $V_{\mathrm{n}}=-16.0 \mathrm{mV}$ & $V_{\mathrm{S}}=-35 \mathrm{mV}$ \\
\hline \hline
\end{tabular}

with the gating variables for $m, n$, and $S$ representing the opening probabilities of the fast and slow potassium channels

$$
\omega_{\infty}(V)=\left[1+\exp \frac{V_{\omega}-V}{\theta_{\omega}}\right]^{-1}, \quad \omega=m, n, S .
$$

Table I lists the parameter values corresponding to the observed bursting dynamics for the model (1). In principle, the characteristic time constant for the membrane potential is determined by the membrane capacity and the corresponding electrical conductance. In accordance with the original formulation of the model, ${ }^{20}$ there is no electrical capacitance in Eq. (1), and the conductances are all dimensionless. To eliminate the dependence on cell size, the conductances have thus been scaled relative to some appropriate conductance. With time constants of $\tau=0.02 \mathrm{~s}$ and $\tau_{S}=35 \mathrm{~s}$, the ratio $k_{S}=\tau / \tau_{S}$ is quite small, and the model is numerically stiff.

Figures 1(a) and 1(b) show typical examples of the time series obtained for the fast variable $V$ and slow variable $S$. Calcium functions as an essential part of a double-sided feedback loop that controls the bursting process and involves modulations of both electrical activity and hormonal secretion. ${ }^{19}$ Calcium removal leads to depolarization and controls the silent phase through deactivation of calcium activated potassium channels. This depolarization activates both the voltage-gated calcium channels and the Hodgkin-Huxley like potassium channels until a renewed influx of calcium takes place and the spiking dynamics is initiated again.

Following Izhikevich, ${ }^{3}$ the bursting attractor in the model (1) is born through simultaneous Hopf and saddlenode bifurcations. As long as $V_{S}<-44.7 \mathrm{mV}$, the equilibrium point is stable [examples of fast and slow manifolds are presented in Fig. 1(d)]. For $V_{S}=-44.7 \mathrm{mV}$, a Hopf bifurcation has occurred, the equilibrium point has turned unstable, and the bursting attractor exists [Fig. 1(e)]. The parameter $V_{S}$ only controls the slow manifold, and with increasing values of this parameter, the slow manifold moves upwards relatively to the fast manifold. The bursting attractor is born in the vicinity of the equilibrium point, but after the bifurcation that occurs for increasing values of $V_{S}$, the equilibrium point moves far away from the bursting attractor. Finally, at $V_{S}=-33.7 \mathrm{mV}$, the bursting attractor turns into the spiking attractor as shown in Fig. 1(f).

An interesting feature of the biological bursting system is the large variation one often observes for the bursting period. We have already referred to this variability in relation to the discussion of slow and fast bursters. Besides variations associated with differences in clusters size, the most obvious reason for variations to occur is inhomogeneity in the cell clusters. This has inspired a number of authors ${ }^{19,20}$ to propose a so-called phantom burster model that allows fast and slow bursting to occur simultaneous, i.e., by replacing the original equation for the slow potassium current by a set of two (or more) parallel potassium currents. The idea has been to use a multiple degree-of-freedom approach to generate a broader range of interacting bursting oscillators while maintaining the overall structure of the system. If the conductance is large, the bursting that results from this feedback will be fast. On the other hand, if the conductance is small, this feedback has little influence, and the bursting that results from it will be slow. In this way, it has been possible
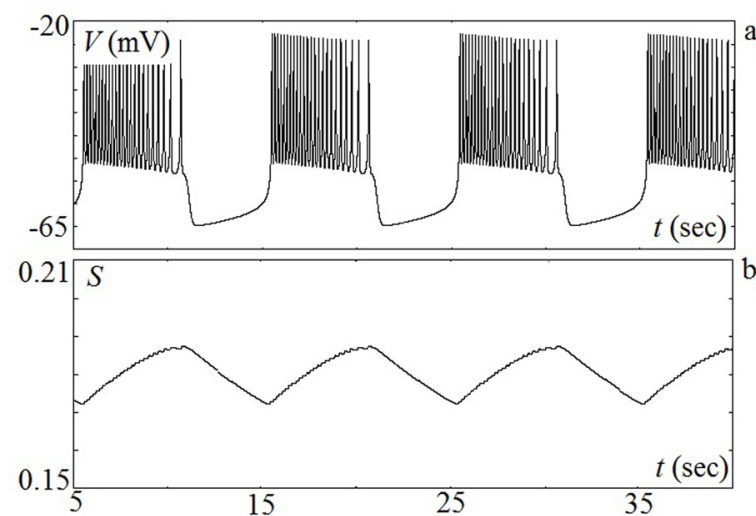

b)
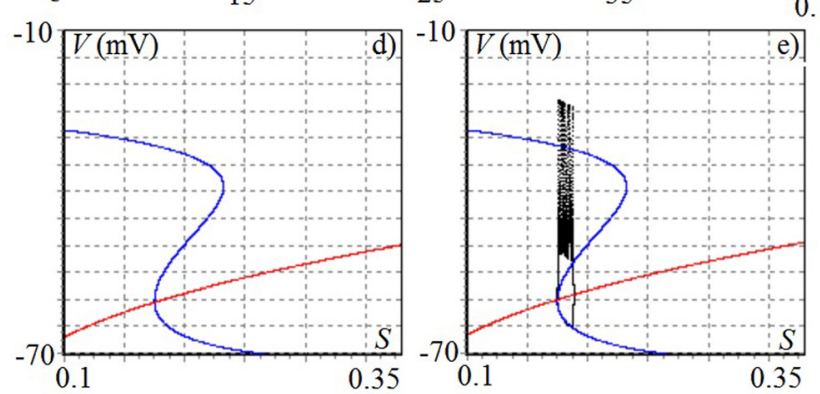

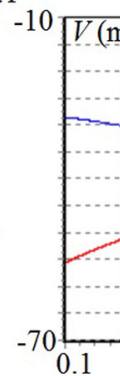

c)
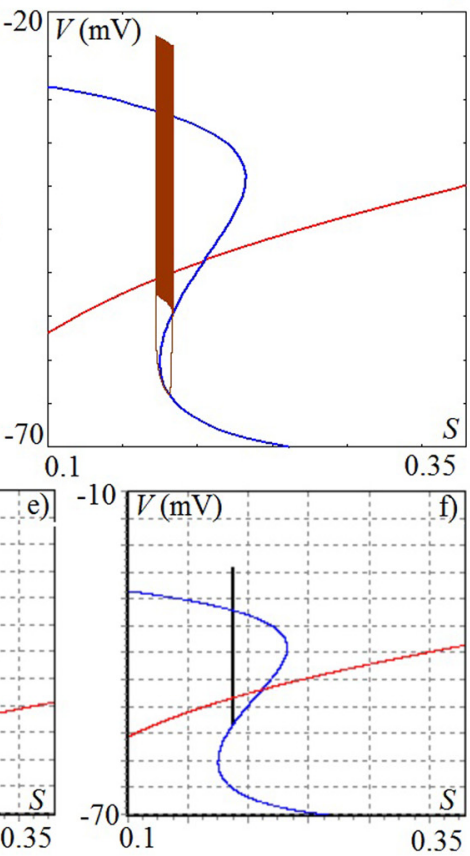

FIG. 1. Time series of the fast (a) and slow (b) variables; (c) fast (blue) and slow (red) manifolds together with a two-dimensional projection of phase portrait for the original Sherman model. ${ }^{18}$ During the spiking phase, $\mathrm{Ca}^{2+}$-ions flow into the cells and, during the silent phases, $\mathrm{Ca}^{2+}$-ions are pumped out. The fast (spiking) dynamics is related to the flow of $\mathrm{K}^{+}$-ions. (d) $V_{S}=-45 \mathrm{mV}$; (e) $V_{S}=-44.7 \mathrm{mV}$; and (f) $V_{S}=-33.7 \mathrm{mV}$. 
to account for a range of biomedical phenomena, including the ubiquitous tri-phase response to a step increase in glucose, the response to perturbations of intra-cellular $\mathrm{Ca}^{2+}$ stores, and different intracellular functions of potassium. ${ }^{20,29}$

\section{MODIFICATION OF THE MODEL}

Figure 1(c) shows the two-dimensional projection of the phase portrait (brown color) together with the fast and slow manifolds for $V_{S}=-35 \mathrm{mV}$. For these parameters, the (periodic) trajectories do not intersect the neighborhood of the equilibrium point, but the bursting state terminates in a homoclinic bifurcation as the trajectory hits the slow manifold at some other point. Hence, it appears possible to reorganize the conditions around the equilibrium point in such a way that this point is stabilized while the bursting trajectory continues to exist. Between the stable equilibrium point and the bursting state, we expect to find some rejecting structure and, under these conditions, the bursting state represents a hidden attractor.

To achieve stabilization of the equilibrium point, we propose a form of the voltage-dependent potassium current that varies strongly with the membrane potential right near this equilibrium point and, hence, its stability can exist without affecting the global flow in the model. The suggested form of the potassium current is specified by the equation:

$$
I_{K 2}(V)=g_{K 2} p_{\infty}(V)\left(V-V_{K}\right),
$$

where the function

$$
p_{\infty}(V)=\left[\exp \frac{V-V_{p}}{\theta_{p}}+\exp \frac{V_{p}-V}{\theta_{p}}\right]^{-1}
$$

represents the opening probability for the suggested new type of potassium channel. The same probability functions for the normal channels are represented by sigmoidal function (5) [see Figs. 2(a) and 2(b)]. When the membrane voltage reaches a threshold voltage, the potassium channel will open with probability $n_{\infty}(V)=1.0$. For the new channels, the opening function never equals to 1.0. When the membrane voltage reaches a threshold voltage, the opening probability will be equal only to 0.5 [this probability function is presented in Fig. 2(c)]. From the physiological point of view, such situation can be interpreted as some dysfunction of ion channel, for instance, like blocking of potassium channel or inactivation. ${ }^{33}$ Also we have to remind that for the individual potassium channels of pancreatic beta-cell the opening probability is very small. ${ }^{21,22}$

Thus, the modified model has the form

$$
\begin{aligned}
\tau \dot{V} & =-I_{C a}(V)-I_{K}(V, n)-I_{K 2}(V)-I_{S}(V, S), \\
\tau \dot{n} & =\sigma\left(n_{\infty}(V)-n\right), \\
\tau_{S} \dot{S} & =S_{\infty}(V)-S,
\end{aligned}
$$

with $I_{K 2}(V)$ and $p_{\infty}(V)$ as given by (6) and (7).

All the parameters of the original model (1) still apply. However, the modified model has three additional parameters $g_{K 2}, V_{p}$, and $\theta_{p}$, which can be used to characterize the new ion channel. Figure 2(e) shows the null-clines of the modified model (8). These curves illustrate how one can

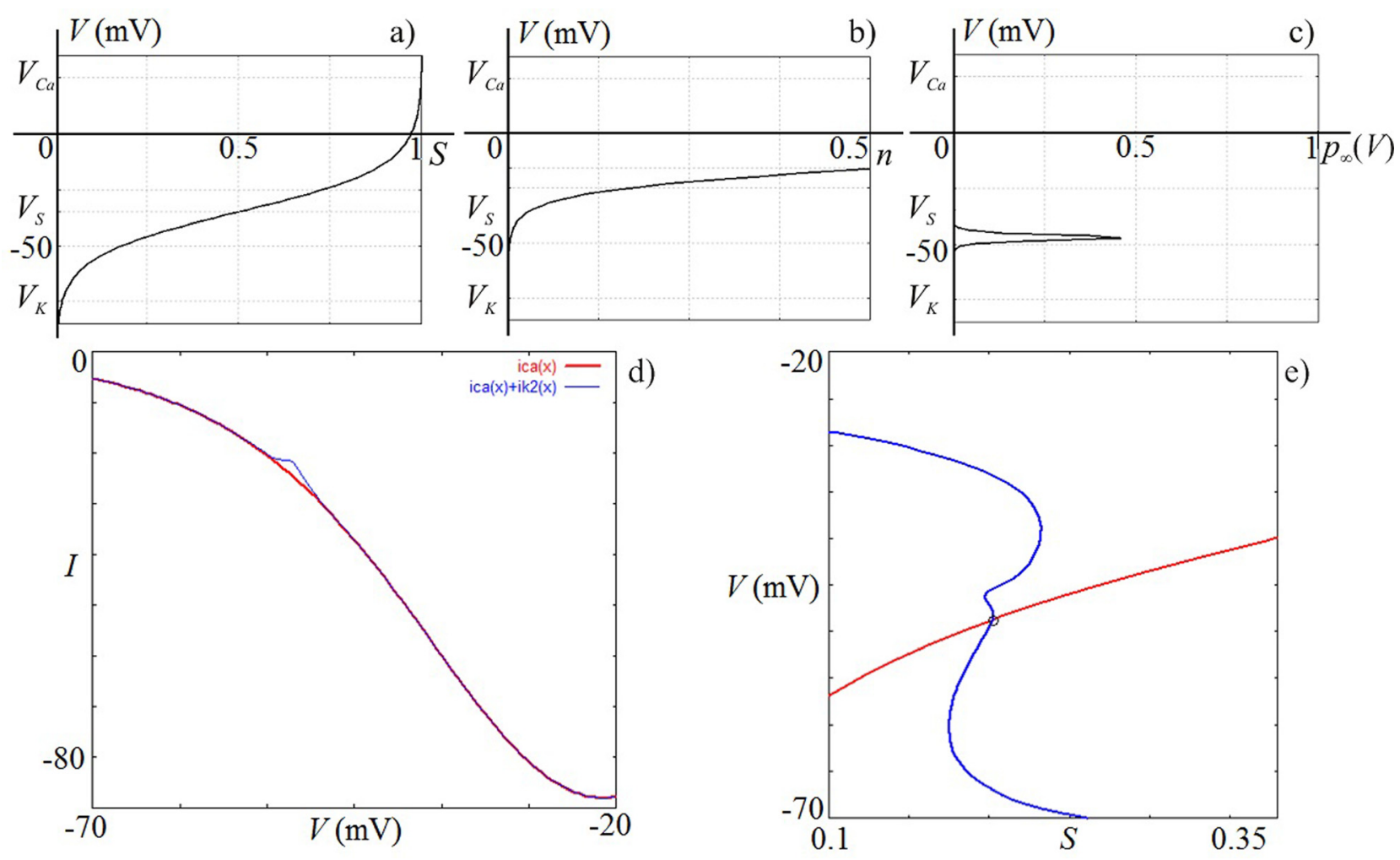

FIG. 2. Dependence of the membrane potential on the different ions: (a) calcium channel; (b) potassium channel; (c) probability function of new ion channel; (d) current of $\mathrm{Ca}^{2+}$ (2) (red) and sum of current $\mathrm{Ca}^{2+}$ (2) and current of new channel (6) (blue); (e) fast (blue) and slow (red) manifolds of the modified model (8). Supplementing parameters for the new ion channel: $g_{K 2}=0.14, \theta_{p}=1 \mathrm{mV}$, and $V_{p}=-46 \mathrm{mV}$. 
introduce new types of ion channels to the model, each leading to the appearance of new pairs of extreme points (minima and maxima) on the fast manifold without affecting the slow manifold. By changing the parameter $V_{p}$, one can determine the extreme points or the range of voltage where the system is most sensitive. The parameter $\theta_{p}=1$ controls the voltage range in which the new ion channel is active in dependence of the membrane potential $V$. In this situation, the equilibrium point can be stable, but bursting dynamics can develop on the same branch of manifold as in the original model (1). Figure 2(d) illustrates the variation of $\mathrm{Ca}^{2+}$ current (red) and of the sum of the $\mathrm{Ca}^{2+}$-currents and the current associated with new ion channel. One can see that, however, changing of the current can only be local, very small, and without major changes of the system. Figure 2(e) shows the fast (blue) and slow (red) manifolds of the modified Sherman model (8).

\section{DYNAMICAL REGIMES}

Let us now consider the mode distribution across a plane spanned by the parameters $V_{p}$ and $g_{K 2}$ that characterize the new ion channel in the model. Figure 3 shows charts of dynamical modes for the modified system (8) with different initial conditions and different magnifications. These charts were constructed in the following way: Using a standard Poincaré section technique, the parameter plane was scanned in small steps. The dynamical state was determined by using a sufficiently long transient for each point in the Poincaré section defined by $n=0.02$. The distribution of points in the Poincaré section and the corresponding number/colors in Fig. 3 are shown below. If the number of points exceeded 120 , the corresponding region was considered to represent a chaotic mode. Charts (a) and (b) were constructed with different initial conditions. Chart (c) is a magnified part of chart (b). In Figs. 3(a) and 3(b), the line of Hopf bifurcation of the equilibrium point is indicated by blue color. This curve was obtained by the use of the software package XPP AUTO.

As the control parameters, we have used the parameter $g_{K 2}$ that characterizes the conductance of the new ion channel and parameter $V_{p}$ that determines the point on the fastslow manifold where the transition occurs. By varying $V_{p}$, we can move the fast manifold relatively to the slow manifold, and in this way we can shift the equilibrium point. In the original model, the unstable equilibrium point falls in the point $\mathrm{EP}_{0}\left(V_{0}, n_{0}, S_{0}\right)=(-48.578,0.0029663,0.2046)$. In the following, we will vary parameter $V_{p}$ in the vicinity of $V_{0}$. In Fig. 3, the vertical green line is the line that corresponds to line $V_{p}=V_{0}$.

For small values of the conductance $g_{K 2}=(0-0.05)$, one observes a broad range with different shades of purple. This color represents bursting dynamics in our model. Different shades of purple reflect different number of spikes per burst. For vanishing supply of $g_{K 2}$, one can see bursting regime with a few spikes per burst. This implies that the influence of the new ion channel on the dynamic of the model (8) is still of limited significance, and the overall dynamics is practically similar to that of the original model (1). When parameter $g_{K 2}$ increases, one can observe an increasing number of spikes per burst. The equilibrium point may become unstable under such variations.

Finally, to the left in the chart of modes, where $g_{K 2}>0.05$, one observes a cascade of period-doubling bifurcations. Green represents a limit cycle of period-1. Period doubling bifurcations take place with decreasing parameter $g_{K 2}$ and increasing parameter $V_{p}$. This dynamical regime represents only spiking dynamics. Figure 4(a) shows the corresponding bifurcation diagram. With increasing values of the parameter $V_{p}$, the spiking limit cycle is transformed into a period- 2 cycle via a period-doubling bifurcation. In the chart of modes, we can hereafter follow the mode lines for the period-doubling bifurcations. Figures 4(b)-4(e) show the examples of spiking attractors, and via projections on the plane $(S, V)$, we can follow how the equilibrium point moves and how the spiking attractor is placed in relation to the form of the fast-slow manifolds. With the increasing values of parameter $V_{p}$, the fast manifold moves upwards relatively to slow manifold, and equilibrium point is shifted into a position between the two extrema.

Lines of period-doublings converge onto individual points located in a corner of the area of a stable equilibrium point. Such regions of parameter space are characteristic for the formation of bursting dynamics associated with a blue sky catastrophe. ${ }^{34}$ A similar phenomenon was observed in a model of a heart interneuron from the medical leech. ${ }^{34} \mathrm{We}$ should also notice that the formation of bursting attractor according to this type of scenario is possible only for values of parameter $V_{p}$ less than $V_{0}\left(V_{p}<V_{0}\right)$.
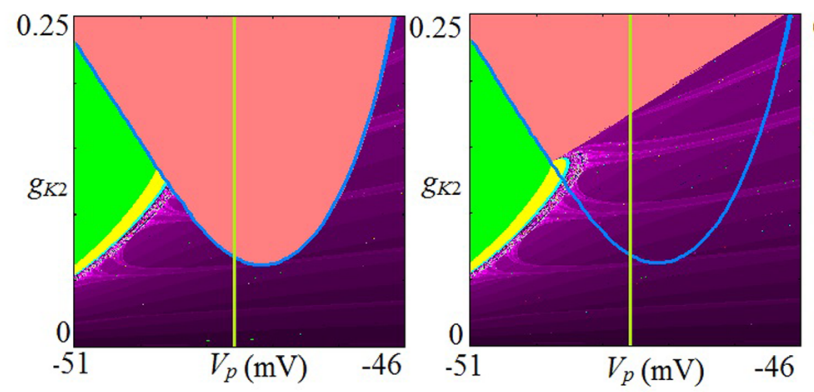

Periodic oscillations $\begin{array}{llllll}1 & 2 & 3 & 4 & 5\end{array}$

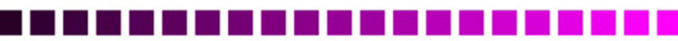
$678891011121314151617181920212223242526-119$

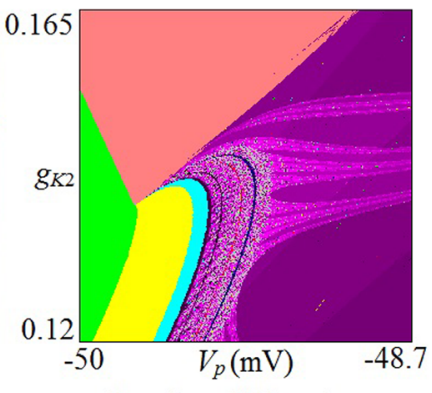

line of Hopf bifurcation

- stable equilibrium point

- chaotic oscillations
FIG. 3. Charts of dynamical modes for the modified model (8). All parameters from Table I remain unchanged and $\theta_{p}=1 \mathrm{mV}$. Initial conditions for charts: (a) $V_{0}=-50 \mathrm{mV}, n_{0}=0.002$, and $S_{0}$ $=0.1984$ and (b) $V_{0}=-40 \mathrm{mV}, n_{0}$ $=0.02$, and $S_{0}=0.1$. (c) Magnified part of Fig. 3(b). In part of the region above the Hopf bifurcation (blue curve), the system displays coexistence of the stable equilibrium point and a variety of periodic and chaotic solutions. 


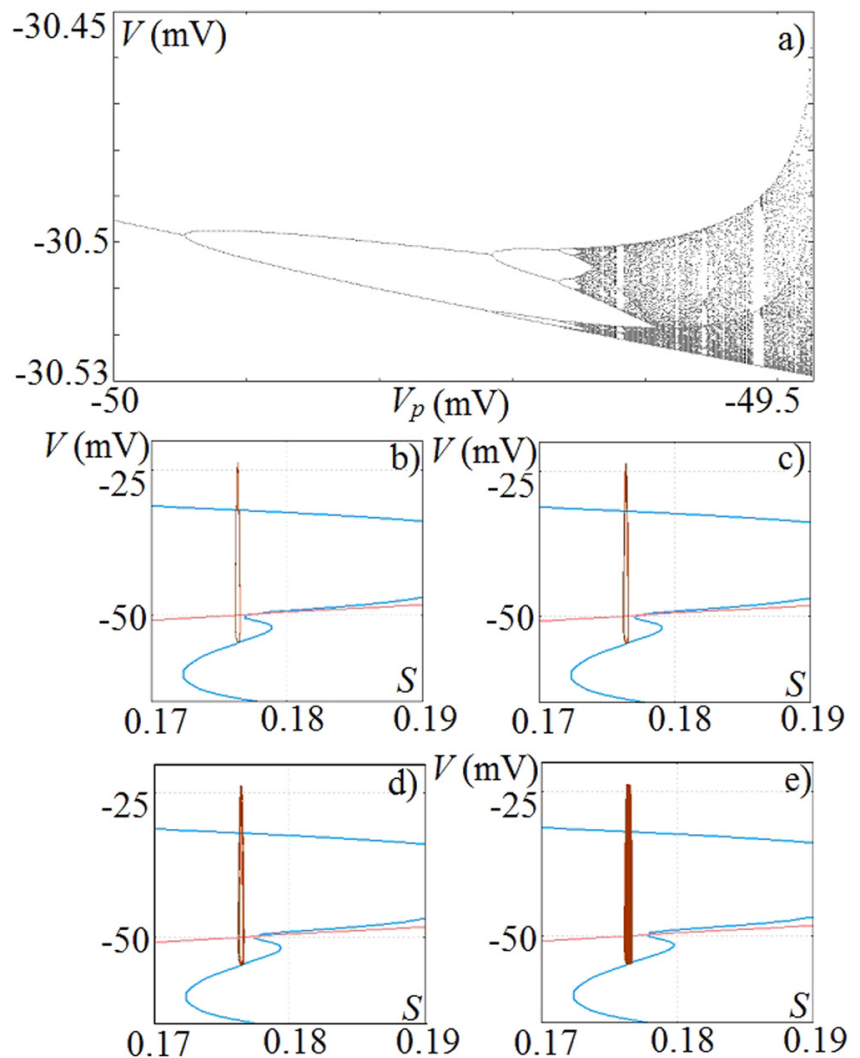

FIG. 4. (a) Bifurcation diagram for the modified model (8) for $g_{K 2}=0.12$ and $\theta_{p}=1$. Two-dimensional projections of phase portraits on the $V-S$ plane demonstrating the transition to chaos through period doubling bifurcations. Only spiking oscillations occur: $g_{K 2}=0.12$ and $\theta_{p}=1$. (b) $V_{p}=-50 \mathrm{mV}$; (c) $V_{p}=-49.9 \mathrm{mV}$; (d) $V_{p}=-49.7 \mathrm{mV}$; and (e) $V_{p}=-49.5 \mathrm{mV}$.

On the right hand side of the chart of modes $\left(V_{p}>V_{0}\right)$, one can observe a broad region with different shades of purple representing bursting dynamics. The bifurcation diagram for the bursting and spiking areas is depicted in Fig. 5(a), and Figs. 5(b) and 5(c) show examples of phase portraits for this form of regime. On the fast-slow manifold, we can see that, when the equilibrium point moves to the maximum, the upper branch of fast manifold becomes unstable, the attractor is shifted to the lower branch, and a long period of oscillations occur. As mentioned above, this transition from spiking to bursting dynamics is associated with a blue-sky catastrophe that occurs at $V_{p} \approx-49.47 \mathrm{mV}$. This transition take place in accordance with the description presented by Shilnikov and Cymbalyuk. ${ }^{34}$ For such kind transformation of the dynamics, the multistability with silent state is possible for larger values of parameter $g_{K 2}$, but there is no coexistence between spiking and bursting.

The bifurcation diagram in Fig. 4(a) is a magnified part of the diagram in Fig. 5(a). Due to the transition associated with blue-sky catastrophe, the areas of spiking and bursting have different scales in respect to variable $V$. In the simplest version, a blue sky catastrophe occurs in three-dimensional phase space. At the bifurcation, a saddle-node limit cycle takes place, whose two-dimensional unstable manifold returns to the periodic orbit making infinitely many rotations in the node region. With a shift of a value of the control parameter in one direction, the saddle-node cycle disappears
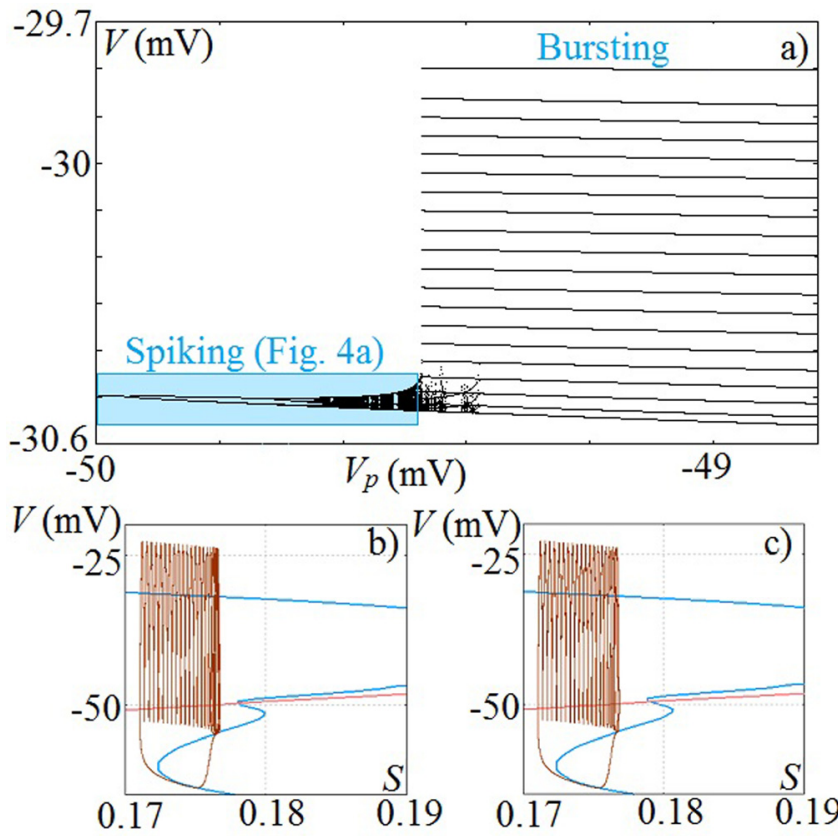

FIG. 5. (a) Bifurcation diagram for the modified model (8) for $g_{K 2}=0.12$ and $\theta_{p}=1$. Two-dimensional projections of phase portraits on the $V$ - $S$ plane demonstrating the transition from chaos to stable bursting dynamics. (b) $V_{p}=-49.4 \mathrm{mV}$ and (c) $V_{p}=-49 \mathrm{mV}$.

and a long large-scale stable periodic orbit containing helical coils near the former saddle-node cycle is born. In Fig. 5(b), one can see helical coils on the place of spiking attractors.

With further increase of parameter $g_{K 2}$, one can see line of Hopf bifurcation. This line corresponds to stabilization of equilibrium point. In Sec. V, we describe in detail the formation of coexistence of regime bursting and regime of silent.

\section{COEXISTING (HIDDEN) ATTRACTORS}

Let us now focus on the coexistent regimes of bursting and of silence (stable equilibrium) as they appear in our modified model. In the charts of dynamical modes (Fig. 3), the red area, representing a region of stable equilibrium, and the purple area, representing a region bursting dynamics, overlap. Hence, depending on the initial conditions, either the stable equilibrium point or the bursting oscillator may be the final state.

In Fig. 6(a), we have plotted the trajectories for a pair of coexisting attracting states in three-dimensional phase space. The purple trajectory represents the stable bursting attractor and the pink curve represents a phase space trajectory that goes to the silent regime. Hence, we conclude that the bursting oscillator (for a range of initial conditions) can operate with a stable equilibrium state in its middle.

Figure 6(b) shows a two-dimensional section of the basin of attraction for the attractors depicted in Fig. 6(a). The section covers the $\left(S_{0}, V_{0}\right)$ plane while the third variable $n_{0}$ was fixed near the equilibrium point at $n_{0}=0.00275$. The dynamical regimes were obtained by Poincare section at the plane $n_{0}=0.02$. Red color represents initial conditions that lead to the stable equilibrium point and purple represents initial conditions that lead to the bursting state. The black curves in Fig. 6(b) represent the lines of the fast and slow 

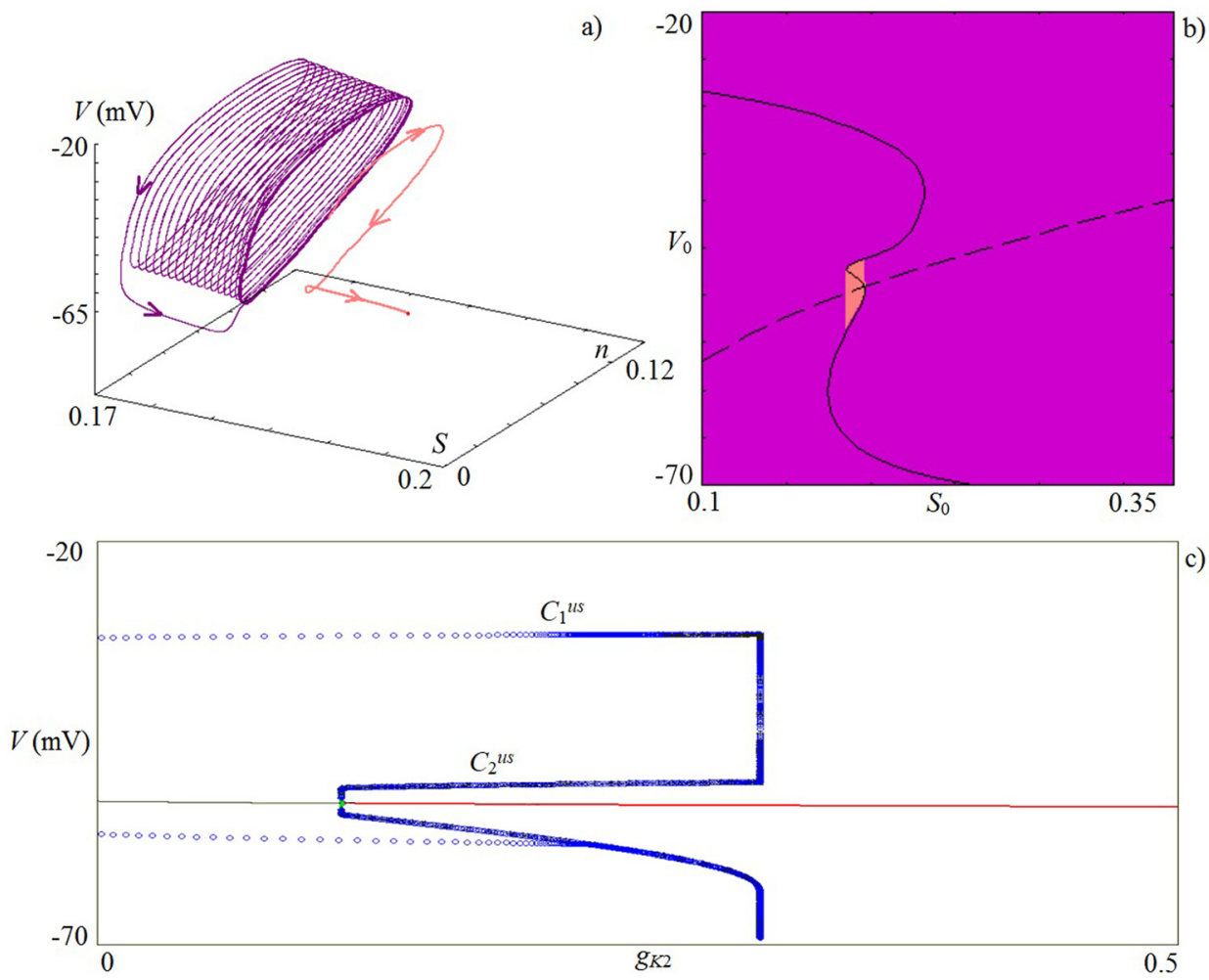

FIG. 6. (a) Three dimensional phase portrait of the modified model (8), $g_{K 2}$ $=0.2, V_{p}=-47 \mathrm{mV}$, and $\theta_{p}=1$. The figure shows the co-existence of the bursting dynamics and the stable equilibrium point; purple and red trajectories have different initial conditions: red $\left[V_{0}=-40 \mathrm{mV}, \quad n_{0}=0.02, \quad\right.$ and $\left.S_{0}=0.187\right]$ and purple $\left[V_{0}=-40 \mathrm{mV}\right.$, $\left.n_{0}=0.02, \quad S_{0}=0.181\right]$; (b) basin of attraction for co-existing bursting attractor (purple) and stable equilibrium point (red), $g_{K 2}=0.2, V_{p}=-47 \mathrm{mV}, \theta_{p}=1$, and $n_{0}=0.00275$; (c) bifurcation diagram in dependence on the parameter $g_{K 2}, V_{p}=-47 \mathrm{mV}$. manifolds. In this way, we can demonstrate that the basin of attraction for the bursting oscillator surrounds a threedimensional region in which the dynamics is controlled by the stable equilibrium point. This island of stable equilibrium dynamics is located between two extrema of null-clines, which occur as a result of the introduction of the new ion channel.

We suppose that the boundary of the basin of attraction for the stable equilibrium point involves an unstable limit cycle together, presumably, with other structures. In order to detect this unstable cycle, we have used numerical bifurcation analysis. In Fig. 6(c), the bifurcation diagram is obtained with XPP AUTO using $g_{K 2}$ as a bifurcation parameter. Red and black lines mark stable and unstable equilibrium point. Blue and green circles mark stable and unstable autooscillations. As we can see from Fig. 6(c), at small values of $g_{K 2}$, there is an unstable equilibrium point together with an unstable cycle $\left(C_{2}{ }^{u s}\right)$.

This unstable cycle associates with the bursting attractor of the original model. At small values of the conductivity $g_{K 2}$, the influence of new ion channel is insignificant and does not change the dynamic of the model. At $g_{K 2}=0.1137$, this point is stabilized as a result of Hopf bifurcation. This bifurcation is supercritical, and the result of bifurcation is the birth of an unstable and stable limit cycle birth in the vicinity of equilibrium point, but hereafter limit cycle became unstable $\left(C_{1}{ }^{u s}\right)$. Thus, the island of regime of silent is limited by unstable cycle $\left(C_{1}{ }^{u s}\right)$, which occur as a result of Hopf bifurcation by adding the new ion channel, but another unstable orbit $\left(C_{2}{ }^{u s}\right)$ which correspond to original bursting attractor persists. At certain value of the parameter $g_{K 2}$ $\left(g_{K 2} \approx 0.3\right)$, a collision of the two unstable orbits take place to leave only a regime of silent.

\section{CONCLUSIONS}

The qualitative theory of dynamical systems provides a rigorous description of the scenarios that produce multistability of regimes in nonlinear dynamical systems. Early studies by Rinzel ${ }^{35}$ and by Guttman et al. ${ }^{36}$ have formulated and answered a number of questions that describe the basic scenario of bistability between tonic spiking and silence. The answer provided is based on the presence of a repelling periodic orbit separating the basin of attraction of the tonic spiking periodic orbit from the state of equilibrium that represents the silent regime. The same scenario also describes the modulation of the neuron dynamics in response to the variations of a bifurcation parameter. According to this scenario, the unstable limit cycle emerges through a subcritical Andronov-Hopf bifurcation and disappears through a saddle-node bifurcation for periodic orbits. These bifurcations define the boundaries of bistability.

In this paper, we have used a modified well-known biophysical model, which describe dynamics of pancreatic betacells to demonstrate the coexistence of a bursting regime and a silent regime. For the considered modification of the model, this type of bistability occurs at the introduction of an additional voltage-dependent potassium current that is activated in the region around the original unstable equilibrium point. The mechanism of bistability is associated with the birth of an unstable cycle as the result of a subcritical Hopfbifurcation inside the bursting attractor. From the point of view of hidden attractors, the bursting regime is a hidden attractor that cannot be reached from initial conditions in the vicinity of the equilibrium point. At the same time, we have shown that the transition from spiking dynamics to bursting dynamics occurs as a result of a blue sky catastrophe. 


\section{ACKNOWLEDGMENTS}

N.V.S. acknowledges the partial support from the Russian Science Foundation under Grant No. 14-21-00041.

${ }^{1}$ P. Heyward, M. Ennis, A. Keller, and M. T. Shipley, "Membrane bistability in olfactory bulb mitral cells," J. Neurosci. 21(14), 5311-5320 (2001); available at http://www.jneurosci.org/content/21/14/5311.full.

${ }^{2}$ Y. Loewenstein, S. Mahon, P. Chadderton, K. Kitamura, H. Sompolinsky, Y. Yarom, and M. Häusser, "Bistability of cerebellar Purkinje cells modulated by sensory stimulation," Nat. Neurosci. 8(2), 202-211 (2005).

${ }^{3}$ E. M. Izhikevich, "Neural excitability, spiking and bursting," Int. J. Bifurcation Chaos 10(06), 1171-1266 (2000).

${ }^{4}$ T. Malashchenko, A. Shilnikov, and G. Cymbalyuk, "Six types of multistability in a neuronal model based on slow calcium current," PloS One 6(7), e21782 (2011).

${ }^{5}$ A. N. Pisarchik and U. Feudel, "Control of multistability," Phys. Rep. 540, 167-218 (2014).

${ }^{6}$ J. R. Pomerening, E. D. Sontag, and J. E. Ferrell, "Building a cell cycle oscillator: Hysteresis and bistability in the activation of Cdc2," Nat. Cell Biol. 5(4), 346-351 (2003).

${ }^{7}$ K. Tsaneva-Atanasova, C. L. Zimliki, R. Bertram, and A. Sherman, "Diffusion of calcium and metabolites in pancreatic islets: Killing oscillations with a pitchfork," Biophys. J. 90(10), 3434-3446 (2006).

${ }^{8}$ D. K. Wells, W. L. Kath, and A. E. Motter, "Control of stochastic and induced switching in biophysical networks," Phys. Rev. X 5, 031036 (2015).

${ }^{9}$ B. N. Kholodenko, "Cell-signalling dynamics in time and space," Nat. Rev. Mol. Cell Biol. 7(3), 165-176 (2006).

${ }^{10} \mathrm{P}$. A. Tass, Phase Resetting in Medicine and Biology: Stochastic Modelling and Data Analysis (Springer Science \& Business Media, 2007).

${ }^{11}$ J. G. Milton, "Epilepsy as a dynamic disease: A tutorial of the past with an eye to the future," Epilepsy Behav. 18(1), 33-44 (2010).

${ }^{12}$ G. Buzsáki and A. Draguhn, "Neuronal oscillations in cortical networks," Science 304(5679), 1926-1929 (2004).

${ }^{13}$ H. Bergman, A. Feingold, A. Nini, A. Raz, H. Slovin, M. Abeles, and E. Vaadia, "Physiological aspects of information processing in the basal ganglia of normal and parkinsonian primates," Trends Neurosci. 21(1), 32-38 (1998).

${ }^{14}$ J. Sarnthein, A. Morel, A. Von Stein, and D. Jeanmonod, "Thalamic theta field potentials and EEG: High thalamocortical coherence in patients with neurogenic pain, epilepsy and movement disorders," Thalamus Relat. Syst. 2(03), 231-238 (2003).

${ }^{15}$ A. Koseska, E. Volkov, and J. Kurths, "Oscillation quenching mechanisms: Amplitude vs. oscillation death," Phys. Rep. 531(4), 173-199 (2013).

${ }^{16}$ G. A. Leonov and N. V. Kuznetsov, "Hidden attractors in dynamical systems. From hidden oscillations in Hilbert-Kolmogorov, Aizerman, and Kalman problems to hidden chaotic attractor in Chua circuits," Int. J. Bifurcation Chaos 23(1), 1330002 (2013).

${ }^{17}$ G. A. Leonov, N. V. Kuznetsov, and T. N. Mokaev, "Homoclinic orbits, and self-excited and hidden attractors in a Lorenz-like system describing convective fluid motion,” Eur. Phys. J. Spec. Top. 224, 1421 (2015).
${ }^{18}$ D. Dudkowski, S. Jafari, T. Kapitaniak, N. V. Kuznetsov, G. A. Leonov, and A. Prasad, "Hidden attractors in dynamical systems," Phys. Rep. 637, $1-50$ (2016)

${ }^{19}$ T. R. Chay and J. Keizer, "Minimal model for membrane oscillations in the pancreatic beta-cell,” Biophys. J. 42(2), 181-189 (1983).

${ }^{20}$ A. Sherman, J. Rinzel, and J. Keizer, "Emergence of organized bursting in clusters of pancreatic beta-cells by channel sharing," Biophys. J. 54(3), 411-425 (1988).

${ }^{21}$ P. Smolen, J. Rinzel, and A. Sherman, "Why pancreatic islets burst but single beta cells do not. The heterogeneity hypothesis," Biophys. J. 64(6), 1668-1680 (1993).

${ }^{22}$ E. Gylfe, E. Grapengiesser, and B. Hellman, "Propagation of cytoplasmic $\mathrm{Ca}^{2+}$ oscillations in clusters of pancreatic $\beta$-cells exposed to glucose," Cell Calcium 12(2-3), 229-240 (1991).

${ }^{23}$ E. Mosekilde, B. Lading, S. Yanchuk, and Y. Maistrenko, "Bifurcation structure of a model of bursting pancreatic cells," BioSystems 63(1), 3-13 (2001).

${ }^{24}$ B. Lading, E. Mosekilde, S. Yanchuk, and Y. Maistrenko, "Chaotic synchronization between coupled pancreatic $\beta$-cells," Prog. Theor. Phys. Suppl. 139, 164-177 (2000).

${ }^{25}$ D. E. Postnov, O. V. Sosnovtseva, S. Y. Malova, and E. Mosekilde, "Complex phase dynamics in coupled bursters," Phys. Rev. E 67(1), 016215 (2003).

${ }^{26}$ A. P. Kuznetsov, S. P. Kuznetsov, E. Mosekilde, and N. V. Stankevich, "Co-existing hidden attractors in a radio-physical oscillator system," J. Phys. A: Math. Theor. 48(12), 125101 (2015).

${ }^{27}$ M. G. Pedersen, E. Mosekilde, K. S. Polonsky, and D. S. Luciani, "Complex patterns of metabolic and $\mathrm{Ca}^{2+}$ entrainment in pancreatic islets by oscillatory glucose,” Biophys. J. 105(1), 29-39 (2013).

${ }^{28}$ T. R. Chay, "On the effect of the intracellular calcium-sensitive $\mathrm{K}+$ channel in the bursting pancreatic beta-cell," Biophys. J. 50(5), 765-777 (1986).

${ }^{29}$ E. Heart and P. J. Smith, "Rhythm of the $\beta$-cell oscillator is not governed by a single regulator: Multiple systems contribute to oscillatory behavior," Am. J. Physiol.-Endocrinol. Metab. 292(5), E1295-E1300 (2007).

${ }^{30}$ G. R. Monteith, D. McAndrew, H. M. Faddy, and S. J. Roberts-Thomson, "Calcium and cancer: Targeting $\mathrm{Ca}^{2+}$ transport," Nat. Rev. Cancer 7(7), 519-530 (2007).

${ }^{31} \mathrm{X}$. Huang and L. Y. Jan, "Targeting potassium channels in cancer," J. Cell Biol. 206(2), 151-162 (2014).

${ }^{32}$ A. Litan and S. A. Langhans, "Cancer as a channelopathy: Ion channels and pumps in tumor development and progression," Front. Cell. Neurosci. 9, 86 (2015).

${ }^{33}$ B. A. Simms and G. W. Zamponi, "Neuronal voltage-gated calcium channels: Structure, function, and dysfunction," Neuron 82(1), 24-45 (2014).

${ }^{34}$ A. Shilnikov and G. Cymbalyuk, "Transition between tonic spiking and bursting in a neuron model via the blue-sky catastrophe," Phys. Rev. Lett. 94(4), 048101 (2005).

${ }^{35}$ J. Rinzel, “On repetitive activity in nerve," Fed. Proc. 37(14), 2793-2802 (1978); available at https://nyuscholars.nyu.edu/en/publications/onrepetitive-activity-in-nerve.

${ }^{36}$ R. Guttman, S. Lewis, and J. Rinzel, "Control of repetitive firing in squid axon membrane as a model for a neuroneoscillator," J. Physiol. 305, 377-395 (1980). 